\title{
AN ANALYSIS OF ALMAGEST MAGNITUDES FOR THE STUDY OF STELLAR EVOLUTION
}

\author{
J. B. HEARNSHAW \\ University of Canterbury, Christchurcy, NZ \\ j.hernshaw@phys.canterbury.ac.nz
}

Visual magnitude data in Ptolemy's Almagest are analysed by comparing them with modern photoelectric magnitudes on the Pogson scale, after taking extinction into account. This result is used to transform Almagest magnitudes to the Pogson scale.

A study is made of changes in the vusual magnitudes of supergiant stars in the Almagest between classical and modern times (an interval of nearly 19 centuries). No evidence is found for any secular changes in the mean brightness of these stars, contrary to the conclusion of Mayer (Observatory $104,77,1984)$. However, it is shown that the Almagest magnitudes for stars in the Milky Way are on average 0.34 Pogson magnitudes too faint and about the Almagest supergiants are affected by this. Finally, some evidence is cited for the visual atmospheric extinction in classical times being significantly less than today. If this is the case, and is not taken into account, it would mimic an apparent supergiant brightening of about 0.1 magnitudes over this time interval. 